\title{
The Influence of Diffuse Left Anterior Descending Artery Lesions on Outcomes of Coronary Artery Bypass Grafting
}

\author{
Yuan-Hsi Tseng, Chih-Chen Kao, Chien-Chao Lin, Ming-Shian Lu, Chu-Hsueh Lu, Yao-Kuang Huang \\ Division of Thoracic and Cardiovascular Surgery, Chia Yi Chang Gung Memorial Hospital, Chia-Yi and Chang Gung University, \\ College of Medicine, Taoyuan, Taiwan
}

\section{ABSTRACT}

Background: Diffuse coronary lesions adversely influence the outcomes of coronary artery bypass grafting (CABG). This study aimed to investigate the influence of diffuse left anterior descending artery (LAD) lesions on the outcomes of CABG.

Methods: The data of 123 patients, who received elective isolated $\mathrm{CABG}$ with the left internal mammary artery bypassed to the LAD from January 2011 to June 2017, were collected. According to their lesions $(\geq 50 \%$ diameter stenoses) $\leq 2 \mathrm{~cm}$ or $>2 \mathrm{~cm}$ in the middle and distal segment of LAD, the patients were classified into a No Diffuse Lesion (NDL) group (69 patients) and a Diffuse Lesion (DL) group (54 patients). The rates of in-hospital mortality, 5-year all-cause mortality, and major cardiac events (MCEs) (i.e. myocardial infarction, angina, acute heart failure, and atrial fibrillation) were analyzed.

Results: According to the univariate analyses, the NDL group had fewer diffuse left circumflex artery (LCX) lesions $(P=.001)$ and higher ventricular fibrillation $(\mathrm{Vf})$ after aortic de-clamping $(P=.03)$ than the DL group. According to the multivariate analyses, the in-hospital and 5-year all-cause mortality rates of the two groups did not significantly differ $(P=.80$ and $P=.59)$. Otherwise, the DL group had a trend toward more MCEs (hazard ratio = 2.07, $P=.061$ ), but the difference clearly was insignificant after adjusting for diffuse LCX lesions and Vf after aortic de-clamping $(P=.104)$.

Conclusions: The results demonstrated that diffuse LAD lesions did not influence the risks of in-hospital mortality, 5-year all-cause mortality, or MCEs after CABG.

\section{INTRODUCTION}

Past studies of patients who underwent coronary artery bypass grafting (CABG) have demonstrated that diffuse coronary lesions not only increased in-hospital mortality

Received March 21, 2020; accepted May 29, 2020.

Correspondence: Yao-Kuang Huang, MD, PhD, Division of Thoracic and Cardiovascular Surgery, Chia-Yi Chang Gung Memorial Hospital, 6 West Section, Chia-Pu Rd, Putzu City, Chiayi County, Taiwan; +886-05-3281200, ext.2864 (e-mail: huang137@icloud.com). rates but also decreased cumulative survival rates after discharge [Graham 1999; Jalal 2007; McNeil 2007]. Moreover, because diffuse atheroma and plaque hinder the sutures of graft-to-coronary artery anastomoses, they may necessitate extra surgical techniques, such as saphenous vein patches and endarterectomy, which may increase the surgical difficulty and the risk of postoperative thrombosis [Byard 1988; Santini 2002; Tasdemir 1996; Walley 1991]. Therefore, we supposed that diffuse left anterior descending artery (LAD) lesions should have a markedly adverse influence on the in-hospital or follow-up outcomes of CABG since the LAD is the largest coronary artery and supplies the majority of blood to the left ventricle. Furthermore, even in cases in which the LAD is bypassed using the left internal mammary artery (LIMA), we inferred that the outcomes of CABG in patients with diffuse LAD lesions should still be inferior to those in patients without diffuse LAD lesions.

This study aimed to compare the postoperative and follow-up outcomes of patients with diffuse

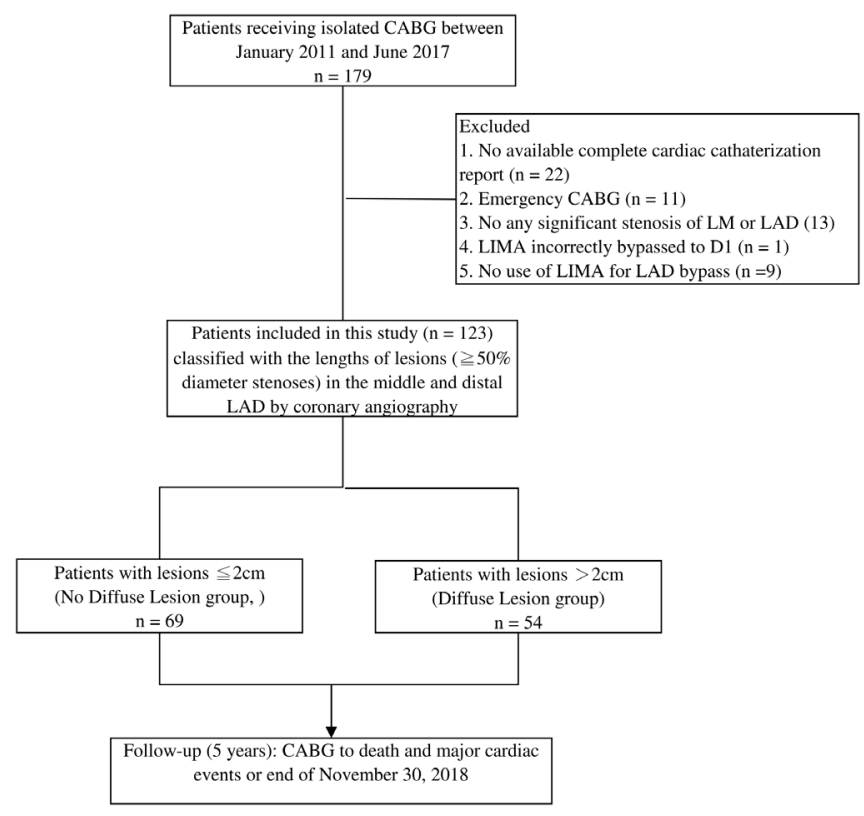

Figure 1. Study diagram. CABG, coronary artery bypass grafting; D1, first diagonal branch; LAD, left anterior descending artery; LIMA, left internal mammary artery; LM, left main coronary artery 
Table 1. Baseline characteristics and preoperative examination results

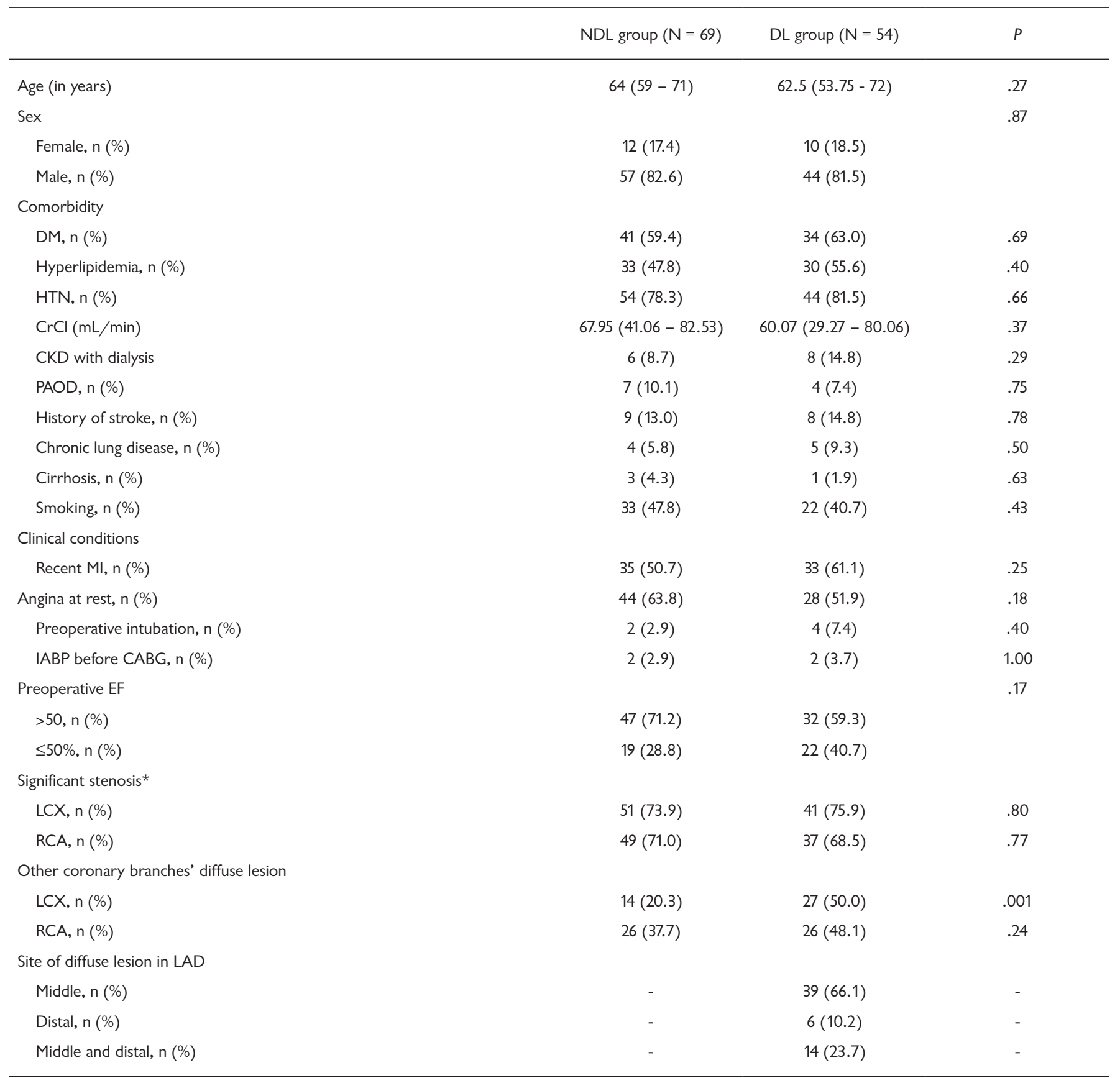

*70\% narrowing of the luminal diameter. CABG, coronary artery bypass grafting; CKD, chronic kidney disease; $\mathrm{CrCl}$, creatinine clearance; DL, diffuse lesion; DM, diabetes mellitus; EF, ejection fraction; HTN, hypertension; IABP, intraaortic balloon pump; LAD, left anterior descending artery; LCX, left circumflex artery; MI, myocardial infarction; NDL, no diffuse lesion; PAOD, peripheral arterial occlusive disease; RCA, right coronary artery

LAD lesions to those of patients without diffuse LAD lesions. However, because a diffuse lesion located in the proximal segment of the LAD is usually not an obstacle to LAD bypass, this study focused on diffuse lesions located in the middle or distal LAD.

\section{MATERIAL AND METHODS}

The protocol for this retrospective study was approved by the Institutional Review Board of Chang Gung Memorial Hospital (CGMF IRB 201900675B0). Because the patient data were retrospective and kept anonymous, 
Table 2. Surgical and cardiopulmonary bypass data

\begin{tabular}{|c|c|c|c|}
\hline Number of grafts & $3(3-3)$ & $3(3-3)$ & .08 \\
\hline RCA territory bypass grafting, $\mathrm{n}(\%)$ & $56(81.2)$ & $49(90.7)$ & .14 \\
\hline TBT (min) & $124(107.5-147)$ & $119(100.75-141.75)$ & .47 \\
\hline CPS (ml) & $4100(2900-5350)$ & $4000(2665-5050)$ & .62 \\
\hline Vf after aortic de-clamping, $\mathrm{n}(\%)$ & $13(18.8)$ & $3(5.6)$ & .03 \\
\hline Difficulty weaning off CPB*, $n(\%)$ & $14(20.3)$ & $13(24.1)$ & .62 \\
\hline
\end{tabular}

\footnotetext{
* need inotropic agent and/or intra-aortic balloon pump counterpulsation to wean off cardiopulmonary bypass. ACT, aortic cross-clamping time; CPB, cardiopulmonary bypass; CPS, cardioplegia solution; DL, diffuse lesion; LCX, left circumflex artery; NDL, no diffuse lesion; RCA, right coronary artery; TBT, total bypass time; Vf, ventricular fibrillation
}

the need for signed informed consent was waived for this study.

This was a retrospective study, using data from a single institution. Data for 179 consecutive patients, who underwent isolated CABG at the institution between January 2011 and June 2017, were initially collected. Of this data, the data for any patients whose cardiac catheterization report was not available or did not have a detailed analysis for the significant lesion were excluded $(\mathrm{N}=22)$ because the interpretation of the coronary angiography was performed by the cardiologists who performed the cardiac catheterization. Furthermore, data for any patients who received an emergency CABG $(\mathrm{N}=11)$, those who did not have any significant stenosis located on the left main coronary artery (LM) or the LAD $(\mathrm{N}=13)$, and those for whom a follow-up coronary angiogram showed that a LIMA was incorrectly bypassed to the first diagonal branch $(\mathrm{N}=1)$ also were excluded. Also, because previous studies proved that the non-use of an internal mammary artery for LAD bypass was a significant predictor of graft occlusion [Dourado 2018; Hillis 2011; Otsuka 2013], data for those patients who did not receive a LIMA-to-LAD bypass were excluded $(N=9)$. Finally, data for a total of 123 patients were included for analysis in this study (Figure 1).

The collected data included each patient's age, sex, comorbidities and preoperative clinical conditions, preoperative examination findings, operative data, surgical outcomes, and all-cause mortality and major cardiac events (MCEs), during follow up after discharge. The MCEs included angina, myocardial infarction, acute heart failure with or without pulmonary edema, and atrial fibrillation.

In this study, the patients were classified into two groups, according to the characteristics of any lesions located in the middle or distal LAD on the coronary angiography, where a lesion was defined as diameter stenosis $\geq 50 \%$ in our hospital [Harris 1980]. Specifically, if there was no lesion or a lesion with a length $\leq 2 \mathrm{~cm}$, the patient was included in the No Diffuse Lesion (NDL) group, whereas if there was a lesion with a length $>2 \mathrm{~cm}$, the patient was included in the Diffuse
Lesion (DL) group. The cut-off point $(2 \mathrm{~cm})$ was determined according to the interpretation of the American College of Cardiology/American Heart Association classification of coronary lesions [Ryan 1988].

In this study, the definition of significant coronary artery stenosis was a finding of $\geq 50 \%$ diameter stenosis for the LM and of $\geq 70 \%$ for other coronary branches [McLaran 1987; Neglia 2015]. The definitions of the LAD's proximal, middle, and distal segments were made according to the coronary artery map published by Alderman et al in 1992 [Alderman 1992]. The definition of acute kidney injury was made according to the RIFLE classification system [Lopes 2013]. Prolonged mechanical ventilation was defined as usage of a mechanical ventilator for more than 21 days [MacIntyre 2005].

Operative method: All of the CABG surgeries were performed via median sternotomy. Great saphenous veins (GSV) were harvested with open methods, including the complete open technique or bridged technique. The left anterior descending artery routinely was bypassed with the LIMA unless it was not available or had poor blood flow. Each surgery was performed with the use of cardiopulmonary bypass with cardiac arrest. The myocardium was protected with cold blood cardioplegia. The anastomosis of graft to coronary arteries was performed with 7-0 Polyprolene. The vein grafts sequentially were connected to non-LAD coronary arteries. Transesophageal echocardiography was used to examine the preoperative and postoperative heart function. Endarterectomy and vein patch for diffuse LAD lesion were seldom performed.

Statistical methods: In the descriptions of the baseline characteristics, preoperative examination results, operative procedures, and operative outcomes, the continuous variables were presented as medians and interquartile ranges, and the categorical variables were presented as numbers and percentages.

In the univariate analyses, comparisons were performed with the Mann-Whitney $U$ test for continuous variables and the Chi-square test or Fisher exact test for categorical variables. 

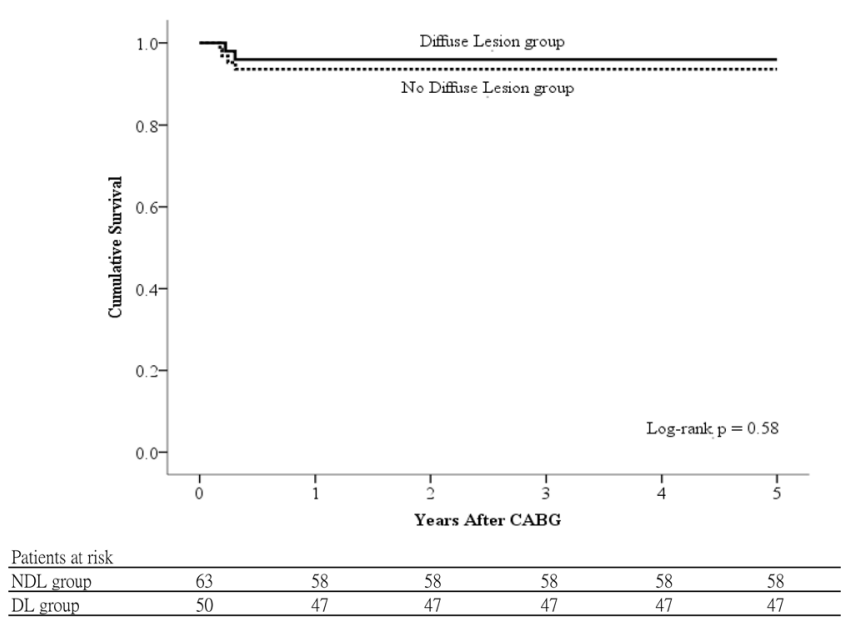

Figure 2. Kaplan-Meier curves of cumulative survival after CABG for the patients who survived to discharge. CABG, coronary artery bypass grafting; DL, diffuse lesion; NDL, no diffuse lesion

In estimating the relevant ratios of in-hospital mortality among the two groups, the odds ratio (OR) that was calculated from the logistic regression was used. The variables that were significantly different between the two groups in the univariate analyses were included into the model.

For the patients who survived to discharge, a survival analysis was conducted using the Kaplan-Meier method and the log-rank test. When estimating the relative risk of allcause mortality and MCEs during follow-up after discharge, the hazard ratio (HR) was calculated with the proportional hazard model. In the survival analysis, the follow-up time was truncated at 5 years or on November 30, 2018, because few cases were left in the study after $>5$ years. Furthermore, when analyzing the risk of MCEs, the patients who died before the occurrence of any MCEs within 5 years were removed to prevent a competitive effect between all-cause mortality and MCEs.

A $P$ value $<.05$ was regarded as statistically significant. Statistical analyses were conducted using SPSS for Windows (Version 17.0; SPSS, Inc., Chicago, IL) and Microsoft Excel 2003.

\section{RESULTS}

Sixty-nine patients were included in the NDL group, and 54 patients were included in the DL group. The baseline characteristics of the patients are shown in Table 1 . There were no statistically significant differences between the two groups, in terms of age, sex, comorbidities, or clinical conditions. In terms of the preoperative examination findings, although the DL group had more patients with a preoperative ejection fraction of $\leq 50 \%$ than the NDL group, the difference between the two groups was not significant $(40.7 \%$ versus $28.8 \%, P=.17$ ). In terms of the coronary angiography results, the DL group had more cases with a diffuse left circumflex artery (LCX) lesion $(50 \%$ versus $20.3 \%, P=.001$ )

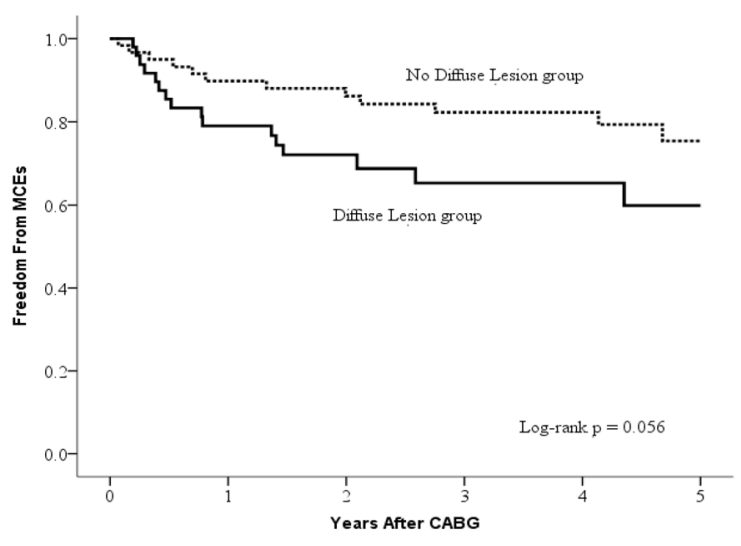

\begin{tabular}{lllllll} 
Patients at risk & \multicolumn{1}{l}{} \\
\hline NDL group & 60 & 52 & 46 & 41 & 31 & 14 \\
\hline DL group & 49 & 35 & 23 & 18 & 14 & 8 \\
\hline
\end{tabular}

Figure 3. Kaplan-Meier curves of cumulative incidence of MCEs after CABG for the patients who survived to discharge. CABG, coronary artery bypass grafting; DL, diffuse lesion; MCEs, major cardiac events; NDL, no diffuse lesion

than the NDL group. Otherwise, a majority of the diffuse LAD lesions in the DL group were located in the middle segment $(66.1 \%)$, followed by the middle to distal segment $(23.7 \%)$, and the distal segment (10.2\%).

According to our surgical database, the DL group had a lower proportion of ventricular fibrillation $(\mathrm{Vf})$ after aortic de-clamping than the NDL group (5.6\% versus $18.8 \%$, $P=.043$ ). However, there were no significant differences, in terms of the number of grafts, LCX territory (including the LCX and obtuse marginal branches) and right coronary territory (including the right coronary artery, posterior descending artery, and posterolateral vessel) bypass grafting, total bypass time, or aortic cross-clamping time (Table 2).

The total overall in-hospital mortality rate was $8.1 \%(\mathrm{~N}$ $=10$ ), and acute myocardial failure was the main cause of in-hospital death $(\mathrm{N}=6,4.9 \%)$. However, the two groups' in-hospital mortality and surgical complication rates did not significantly differ (Table 3).

According to the multivariate analyses, diffuse LAD lesions did not increase the risk of in-hospital mortality $(\mathrm{OR}=$ $0.84, P=.80$ ), and the variables that were significantly different between the two groups in the univariate analyses, which were the rate of diffuse LCX lesions and Vf after aortic declamping, did not influence the association between diffuse LAD lesions and the in-hospital mortality (Table 4).

For the patients who survived to discharge, a Kaplan-Meier analysis of the 5 -year cumulative survival rate did not indicate a statistically significant difference between the groups (logrank $P=.58$ ), but the DL group had more MCEs, and the difference between the two groups in this regard was nearly significant $(\log$-rank $P=.056)$ (Figures 2 and 3). Nonetheless, according to the multivariate analyses, after adjusting for diffuse LCX lesions and Vf after aortic de-clamping, the association between the diffuse LAD lesions and MCEs was not statistically significant (crude HR $=2.05, P=.061$; adjusted $\mathrm{HR}=1.93, P=.104)$. Otherwise, there also was no significant 
Table 3. Postoperative outcomes and complications

\begin{tabular}{|c|c|c|c|}
\hline In-hospital mortality, n (\%) & $6(8.7)$ & $4(7.4)$ & 1.00 \\
\hline ICU stay (day) & $4(3-6)$ & $4(3-6)$ & .57 \\
\hline Hospital stay (day) & $12(9-18)$ & $13.5(10-19)$ & .32 \\
\hline New stroke, n (\%) & $1(1.4)$ & $2(3.7)$ & .58 \\
\hline Myocardial failure, n (\%) & $4(5.8)$ & $4(7.4)$ & .73 \\
\hline Prolonged MV*, n (\%) & $5(7.2)$ & $4(7.4)$ & 1.00 \\
\hline Acute kidney injury\#, n (\%) & $7(10.1)$ & $8(14.8)$ & .58 \\
\hline Sternotomy wound infection, $\mathrm{n}(\%)$ & $3(4.3)$ & $5(9.3)$ & .30 \\
\hline
\end{tabular}

* > 21 days, \# according to RIFLE criteria. DL, diffuse lesion; ICU, intensive care unit; MV, mechanical ventilation; NDL, no diffuse lesion

difference between the two groups, in terms of 5 -year allcause mortality (crude HR $=0.62, P=.59$; adjusted HR = $0.52, P=.46)$ (Table 5).

According to the follow-up coronary angiographies for MCEs after discharge, among the NDL group, there were three patients $(4.76 \%)$ who suffered LIMA-to-LAD total occlusion, but among the DL group, only one patient (2\%) suffered $50 \%$ stenosis in the LIMA-to-LAD graft. Nonetheless, the difference between the two groups in this regard was not statistically significant $(P=.63)$.

\section{DISCUSSION}

Although a diffuse coronary lesion is no longer a powerful predictor of percutaneous coronary intervention (PCI) failure [Hermans 1993; Myler 1992; Tsagalou 2005], previous studies have indicated that such lesions still have adverse impacts on the outcomes of CABG [Graham 1999; Jalal 2007; McNeil 2007]. Many surgical techniques have been proposed, and among those techniques, long LAD arteriotomy with reconstruction with LIMA lay-on patch has been found to have better outcomes [Fukui 2005; Nishi 2005; Zattera 2011]. Nonetheless, this technique requires a learning curve and may prolong the ischemic time and bypass time. In this study, meanwhile, we demonstrated that diffuse LAD lesions did not influence the outcomes even of CABG surgeries performed without special techniques.

Past research has shown that incomplete revascularization of the LAD mainly influences the 5-10 year mortality rather than early postoperative mortality [Dourado 2018; Lawrie 1982; Leviner 2018] because effective side branches or collaterals may compensate for the myocardial perfusion in ischemic heart disease for some period of time [Seiler 2013], after which, with the progression of stenosis [Pereg 2014; Tanaka 2016], the clinical symptoms and signs may deteriorate. In our study, the 5-year follow-up of the all-cause mortality and MCEs did not indicate any significant difference between the two groups in regard to those outcomes. These results were compatible with previous studies that found that the use of LIMA-to-LAD bypass was usually the most important determinant of good outcomes for CABG [Dourado 2018; Hillis 2011; Otsuka 2013]. Nonetheless, according to the hazard ratios yielded by our multivariate analyses, the patients with diffuse LAD lesions had a trend toward a higher rate of MCEs, especially before adjustment. Therefore, a larger sample size would be required to confirm the influence of diffuse LAD lesions on the mid-term and long-term outcomes.

The high rate of in-hospital mortality $(8 \%)$ was one of the main concerns in this study. Past studies have demonstrated that the surgical mortality rate of CABG should be 2-3\% [Ferguson 2002; Hansen 2015; Lamy 2012]. The most probable cause for the relatively high rate in this study is that our hospital is a low-volume hospital, in terms of the number of cardiac surgeries performed (about 80-100 heart surgeries/year) [Carey 2003; Gonzalez 2014]. This suggests that our operating room and intensive care unit staff's technical and care skills may need more time to mature. The evidence most clearly supporting this conclusion is that from January 2011 to November 2015, our in-hospital mortality rate for elective CABG was about $10 \%$, whereas from December 2015 to June 2017, the in-hospital mortality rate was zero. Otherwise, in comparison to patients included in previous studies [Lamy 2012; Shroyer 2009], our patients had higher proportions of end-stage renal disease requiring dialysis $(11.4 \%)$, diabetes mellitus $(61 \%)$, and history of stroke (13.8\%), and these patient-related factors also may explain why our hospital had a relatively high rate of surgical mortality.

According to our surgical data, endarterectomies and vein patches were rarely performed and only performed on coronary branches beyond the LAD, such as obtuse 
Table 4. Odds ratio of diffuse lesion in left anterior descending artery versus in-hospital mortality, with adjustments for diffuse left circumflex artery lesion and ventricular fibrillation after aortic de-clamping

\begin{tabular}{lcc}
\hline & OR & $95 \%$ Cl \\
\hline Crude & Ref & \\
NDL group & 0.84 & $0.23-3.14$ \\
DL group & & .80 \\
Interaction analysis & & $0.15-4.42$ \\
Diffuse LCX lesion & 0.80 & $0.09-12.57$ \\
No & 1.04 & .80 \\
Yes & 0.87 & .98 \\
$P$-value & & $0.17-3.82$ \\
Vf after aortic de-clamping & $0.16-46.79$ \\
No & 0.81 & .79 \\
Yes & 2.75 & $0.25-4.62$ \\
P-value & 0.46 & 0.93 \\
\hline
\end{tabular}

The model was adjusted by diffuse LCX lesion and $\mathrm{Vf}$ after aortic de-clamping. $\mathrm{Cl}$, confidence interval; $\mathrm{DL}$, diffuse lesion; LCX, left circumflex artery; NDL, no diffuse lesion; OR, odds ratio; Vf, ventricular fibrillation

marginal branches $(\mathrm{N}=3$, including one using a vein patch), posterior descending arteries $(\mathrm{N}=2)$, left circumflex arteries $(\mathrm{N}=1)$, and right coronary arteries $(\mathrm{N}=3)$, for diffuse atherosclerotic lesions. The first reason for this is that the vessel walls usually become thin and fragile after endarterectomy, which makes suturing difficult and can easily lead to graft-to-coronary artery anastomosis bleeding. Otherwise, according to our patients' follow-up coronary angiographies, the diffuse LAD lesions did not decrease the patency of LIMA-to-LAD bypass grafting statistically under common anastomotic methods. In past studies, it was also demonstrated that endarterectomy may increase the risk of thrombosis and postoperative mortality [Byard 1988; Tasdemir 1996; Walley 1991]. However, there were no patients who received long-segment LAD reconstruction with LIMA patch in this study, and that technique may be the next step that we should try.

In enrolling patients in the study, we excluded nine patients who did not receive a LIMA to LAD graft to decrease the influence of middle-term outcomes caused by the different patency rates of different grafts. Past studies have reported 5-year and 6-year patency rates of $82 \%$ and $76 \%$, respectively, for GSV grafts [Bourassa 1985; Goldman 2004], while also reporting that LIMA grafts have a 10 -year patency rate $>85 \%$ because of lower rates of intimal hyperplasia and atherosclerosis [Hillis 2011; Goldman 2004; Otsuka 2013]. Since our follow-up duration was truncated at 5 years, excluding those patients for whom the GSV was used to bypass the LAD could have removed a factor that may have affected the survival analysis. Relatedly, although Karthik et al reported that using different kinds of grafts for bypasses to the LAD did not influence in-hospital mortality and morbidity [Karthik 2004], we did not have the same experience. In our surgical data, after excluding the patients who received a GSV to LAD bypass, the total in-hospital mortality was decreased from $11.36 \%$ to $8.1 \%$. The overall in-hospital mortality rate of the nine patients for whom the GSV was used to bypass the LAD was $55.6 \%$, a rate which had a statistically significant difference from that of the patients who received a LIMA to LAD graft $(P=.001)$. However, the rates of death from heart failure for the two groups of patients did not significantly differ $(P=.397)$. These results suggested that the patients for whom the GSV was used to bypass the LAD had more lethal complications than the patients who received a LIMA to LAD graft. These results may be associated with the proficiency of the surgeon and medical team, in terms of surgical practice and postoperative care.

It is hard to explain why our results showed that the patients with diffuse LAD lesions had a lower incidence of $\mathrm{Vf}$ after aortic de-clamping. The mechanism of Vf after aortic de-clamping is unclear and a variety of potential reasons for it have been suggested, such as poor myocardial protection, hypothermia, electrolyte imbalance, and reperfusion injury [Morita 2010; Watanabe 2011]. On the basis of our results, we supposed that the diffuse lesions slowed down the blood flow following de-clamping of the LIMA graft and aorta and resulted in effects that were similar to those of postconditioning, which is a technique used to decrease reperfusion injury and myocardial infarct size after revascularization [Jivraj 2015]. However, the effects of postconditioning themselves are controversial [Hahn 2013; Laskey 2013], so 
Table 5. Hazard ratio of diffuse lesion in left anterior descending artery versus all-cause mortality and major cardiac events over 5 years, with adjustments for diffuse left circumflex artery lesion and ventricular fibrillation after aortic de-clamping

\begin{tabular}{|c|c|c|c|c|c|c|}
\hline & $\mathrm{HR}$ & $95 \% \mathrm{Cl}$ & $P$ & $\mathrm{HR}$ & $95 \% \mathrm{Cl}$ & $P$ \\
\hline NDL group & Ref & & & Ref & & \\
\hline DL group & 0.62 & $0.11-3.40$ & 0.59 & 2.05 & $0.97-4.35$ & 0.061 \\
\hline \multicolumn{7}{|l|}{ Diffuse LCX lesion } \\
\hline No & 0.65 & $0.07-6.25$ & 0.71 & 1.50 & $0.58-3.90$ & 0.40 \\
\hline Yes & 0.50 & $0.03-7.99$ & 0.62 & 6.00 & $0.76-47.51$ & 0.09 \\
\hline$P$-value & 0.89 & & & 0.26 & & \\
\hline \multicolumn{7}{|c|}{$V f$ after aortic de-clamping } \\
\hline Confounding effect $\ddagger$ & 0.52 & $0.09-3.02$ & 0.46 & 1.93 & $0.87-4.26$ & 0.104 \\
\hline
\end{tabular}

*All patients who had ever suffered $\mathrm{Vf}$ after aortic de-clamping were followed up 5 years, and only one patient suffered major cardiac events by 5 years †Major cardiac events including angina, myocardial infarction, acute heart failure (including pulmonary edema), and atrial fibrillation $\ddagger$ The model was adjusted by diffuse LCX lesion and $\mathrm{Vf}$ after aortic de-clamping

$\mathrm{Cl}$, confidence interval; DL, diffuse lesion; HR, hazard ratio; NDL, no diffuse lesion; LCX, left circumflex artery; Vf, ventricular fibrillation

our speculation requires further investigation. Fortunately, the incidences of $\mathrm{V} f$ that we encountered all could be treated with current defibrillation and/or the administration of antiarrhythmic agents.

Limitations: While our sample of patients receiving CABGs was too small to make any generalizations, the results may indicate that diffuse lesions in the LAD can be effectively treated with CABG, especially when using a LIMA for the LAD bypass. Nonetheless, the sample size made it impossible for this study to further discriminate the influence between middle and distal diffuse lesions, which is relevant because past studies have suggested that distal lesions in coronary arteries may have bigger impacts on or even preclude the possibility of CABG. However, we thought this body of research still has undeniable merit in terms of offering valuable insights into surgical decision making.

In order to be sure of the consistency of the surgical methods, we collected surgical data beginning after 2010, a year when there was a transition from old to new cardiac surgeons and changes to part of the surgical team staff. We also excluded the patients whose CABG was performed by the former cardiac surgeon in early 2011. Otherwise, to have an adequate duration of follow up for the survival analysis, we needed to include all the data from after this transition even though doing so may have made our outcomes unsatisfactory. Nonetheless, this should not have influenced the comparison of outcomes between the two groups, since they both received surgery under the same circumstances.

\section{CONCLUSIONS}

Our results demonstrated that diffuse LAD lesions did not influence the outcomes of CABG, including in-hospital mortality, 5-year all-cause mortality, and the occurrence of MCEs. Therefore, CABG should be a feasible treatment even in cases where there is a diffuse LAD lesion. However, further research studies with greater sample sizes still are needed to confirm the outcomes of the present study.

\section{REFERENCES}

Alderman EL, Stadius M. 1992. The angiographie definitions of the Bypass Angioplasty Revascularization Investigation. Coronary Artery Disease. 3(12):1189-1208.

Bourassa MG, Fisher LD, Campeau L, Gillespie MJ, McConney M, Lesperance J. 1985. Long-term fate of bypass grafts: the Coronary Artery Surgery Study (CASS) and Montreal Heart Institute experiences. Circulation. Dec;72(6 Pt 2):V71-78.

Byard RW, Keon WJ, Walley VM. 1988. Coronary endarterectomy: the long-term local effects. The American journal of cardiovascular pathology. 2(1):31-38.

Carey JS, Robertson JM, Misbach GA, Fisher AL. 2003. Relationship of hospital volume to outcome in cardiac surgery programs in California. The American surgeon. Jan;69(1):63-68.

Dourado LOC, Bittencourt MS, Pereira AC, et al. 2018. Coronary Artery Bypass Surgery in Diffuse Advanced Coronary Artery Disease: 
1-Year Clinical and Angiographic Results. The Thoracic and cardiovascular surgeon. Sep;66(6):477-482.

Ferguson TB, Jr., Hammill BG, Peterson ED, DeLong ER, Grover FL. 2002. A decade of change--risk profiles and outcomes for isolated coronary artery bypass grafting procedures, 1990-1999: a report from the STS National Database Committee and the Duke Clinical Research Institute. Society of Thoracic Surgeons. The Annals of thoracic surgery. Feb;73(2):480-489; discussion 489-490.

Fukui T, Takanashi S, Hosoda Y. 2005. Coronary endarterectomy and stent removal in patients with in-stent restenosis. The Annals of thoracic surgery. Feb;79(2):558-563; discussion 563.

Goldman S, Zadina K, Moritz T, et al. 2004. Long-term patency of saphenous vein and left internal mammary artery grafts after coronary artery bypass surgery: results from a Department of Veterans Affairs Cooperative Study. Journal of the American College of Cardiology. Dec 7;44(11):2149-2156.

Gonzalez AA, Dimick JB, Birkmeyer JD, Ghaferi AA. 2014. Understanding the volume-outcome effect in cardiovascular surgery: the role of failure to rescue. JAMA surgery. Feb;149(2):119-123.

Graham MM, Chambers RJ, Davies RF. 1999. Angiographic quantification of diffuse coronary artery disease: reliability and prognostic value for bypass operations. The Journal of thoracic and cardiovascular surgery. Oct;118(4):618-627.

Hahn JY, Song YB, Kim EK, et al. 2013. Ischemic postconditioning during primary percutaneous coronary intervention: the effects of postconditioning on myocardial reperfusion in patients with ST-segment elevation myocardial infarction (POST) randomized trial. Circulation. Oct 22;128(17):1889-1896.

Hansen LS, Hjortdal VE, Andreasen JJ, Mortensen PE, Jakobsen CJ. 2015. 30-day mortality after coronary artery bypass grafting and valve surgery has greatly improved over the last decade, but the 1-year mortality remains constant. Annals of cardiac anaesthesia. Apr-Jun;18(2):138-142.

Harris PJ, Behar VS, Conley MJ, et al. 1980. The prognostic significance of $50 \%$ coronary stenosis in medically treated patients with coronary artery disease. Circulation. Aug;62(2):240-248.

Hermans WR, Foley DP, Rensing BJ, et al. 1993. Usefulness of quantitative and qualitative angiographic lesion morphology, and clinical characteristics in predicting major adverse cardiac events during and after native coronary balloon angioplasty. CARPORT and MERCATOR Study Groups. Am J Cardiol. Jul 1;72(1):14-20.

Hillis LD, Smith PK, Anderson JL, et al. 2011. 2011 ACCF/AHA Guideline for Coronary Artery Bypass Graft Surgery: executive summary: a report of the American College of Cardiology Foundation/American Heart Association Task Force on Practice Guidelines. Circulation. Dec 6;124(23):2610-2642.

Jalal A. 2007. An objective method for grading of distal disease in the grafted coronary arteries. Interactive cardiovascular and thoracic surgery. Aug;6(4):451-455.

Jivraj N, Liew F, Marber M. 2015. Ischaemic postconditioning: cardiac protection after the event. Anaesthesia. May;70(5):598-612.

Karthik S, Srinivasan AK, Grayson AD, Jackson M, Mediratta NK. 2004. Left internal mammary artery to the left anterior descending artery: effect on morbidity and mortality and reasons for nonusage. The Annals of thoracic surgery. Jul;78(1):142-148.

Lamy A, Devereaux PJ, Prabhakaran D, et al. 2012. Off-pump or onpump coronary-artery bypass grafting at 30 days. The New England journal of medicine. Apr 19;366(16):1489-1497.
Laskey WK, Schevchuck A. 2013. Postconditioning during percutaneous coronary intervention in acute myocardial infarction: continued difficulty in translation. Circulation. Oct 22;128(17):1858-1860.

Lawrie GM, Morris GC, Jr., Silvers A, et al. 1982. The influence of residual disease after coronary bypass on the 5-year survival rate of 1274 men with coronary artery disease. Circulation. Oct;66(4):717-723.

Leviner DB, Torregrossa G, Puskas JD. 2018. Incomplete revascularization: what the surgeon needs to know. Ann Cardiothorac Surg. Jul;7(4):463-469.

Lopes JA, Jorge S. 2013. The RIFLE and AKIN classifications for acute kidney injury: a critical and comprehensive review. Clinical kidney journal. Feb;6(1):8-14.

MacIntyre NR, Epstein SK, Carson S, Scheinhorn D, Christopher K, Muldoon S. 2005. Management of patients requiring prolonged mechanical ventilation: report of a NAMDRC consensus conference. Chest. Dec;128(6):3937-3954.

McLaran CJ, Gersh BJ, Sugrue DD, et al. 1987. Out-of-hospital cardiac arrest in patients without clinically significant coronary artery disease: comparison of clinical, electrophysiological, and survival characteristics with those in similar patients who have clinically significant coronary artery disease. British heart journal. Dec;58(6):583-591.

McNeil M, Buth K, Brydie A, MacLaren A, Baskett R. 2007. The impact of diffuseness of coronary artery disease on the outcomes of patients undergoing primary and reoperative coronary artery bypass grafting. European journal of cardio-thoracic surgery: official journal of the European Association for Cardio-thoracic Surgery. May;31(5):827-833.

Morita Y, Mizuno J, Yoshimura T, Morita S. 2010. Efficacy of amiodarone on refractory ventricular fibrillation resistant to lidocaine and cardioversion during weaning from cardiopulmonary bypass in aortic valve replacement for severe aortic stenosis with left ventricular hypertrophy. Journal of anesthesia. Oct;24(5):761-764.

Myler RK, Shaw RE, Stertzer SH, et al. 1992. Lesion morphology and coronary angioplasty: current experience and analysis. Journal of the American College of Cardiology. Jun;19(7):1641-1652.

Neglia D, Rovai D, Caselli C, et al. 2015. Detection of significant coronary artery disease by noninvasive anatomical and functional imaging. Circulation. Cardiovascular imaging. Mar;8(3).

Nishi H, Miyamoto S, Takanashi S, et al. 2005. Optimal method of coronary endarterectomy for diffusely diseased coronary arteries. The Annals of thoracic surgery. Mar;79(3):846-852; discussion 852-843.

Otsuka F, Yahagi K, Sakakura K, Virmani R. 2013. Why is the mammary artery so special and what protects it from atherosclerosis? Ann Cardiothorac Surg. Jul;2(4):519-526.

Pereg D, Fefer P, Samuel M, et al. 2014. Native coronary artery patency after coronary artery bypass surgery. JACC. Cardiovascular interventions. Jul;7(7):761-767.

Ryan TJ, Faxon DP, Gunnar RM, et al. 1988. Guidelines for percutaneous transluminal coronary angioplasty. A report of the American College of Cardiology/American Heart Association Task Force on Assessment of Diagnostic and Therapeutic Cardiovascular Procedures (Subcommittee on Percutaneous Transluminal Coronary Angioplasty). Circulation. Aug;78(2):486-502.

Santini F, Casali G, Lusini M, et al. 2002. Mid-term results after extensive vein patch reconstruction and internal mammary grafting of the diffusely diseased left anterior descending coronary artery. European journal of cardio-thoracic surgery : official journal of the European Association for Cardio-thoracic Surgery. Jun;21(6):1020-1025. 
Seiler C. 2013. Assessment and impact of the human coronary collateral circulation on myocardial ischemia and outcome. Circulation. Cardiovascular interventions. Dec;6(6):719-728.

Shroyer AL, Grover FL, Hattler B, et al. 2009. On-pump versus offpump coronary-artery bypass surgery. The New England journal of medicine. Nov 5;361(19):1827-1837.

Tanaka A, Ishii H, Oshima H, et al. 2016. Progression from stenosis to occlusion in the proximal native coronary artery after coronary artery bypass grafting. Heart and vessels. Jul;31(7):1056-1060.

Tasdemir O, Kiziltepe U, Karagoz HY, Yamak B, Korkmaz S, Bayazit K. 1996. Long-term results of reconstructions of the left anterior descending coronary artery in diffuse atherosclerotic lesions. The Journal of thoracic and cardiovascular surgery. Sep;112(3):745-754.

Tsagalou E, Chieffo A, Iakovou I, et al. 2005. Multiple overlapping drug-eluting stents to treat diffuse disease of the left anterior descending coronary artery. Journal of the American College of Cardiology. May 17;45(10):1570-1573.

Walley VM, Byard RW, Keon WJ. 1991. A study of the sequential morphologic changes after manual coronary endarterectomy. The Journal of thoracic and cardiovascular surgery. Dec;102(6):890-894.

Watanabe G, Yashiki N, Tomita S, Yamaguchi S. 2011. Potassiuminduced cardiac resetting technique for persistent ventricular tachycardia and fibrillation after aortic declamping. The Annals of thoracic surgery. Feb;91(2):619-620.

Zattera GF, D'Armini AM, Vigano M. 2011. Late angiographic changes of the left anterior descending coronary artery following removal of multiple occluded stents and on-lay patching with the left internal mammary artery. European journal of cardio-thoracic surgery : official journal of the European Association for Cardio-thoracic Surgery. Dec;40(6):1529-1530. 